Acta Materialia, Vol. 57, no. 2, pp. 525-532 (2009)

\title{
Multiscale Modeling of Polymer Materials Using a Statistics-Based Micromechanics Approach
}

\author{
P.K. Valavala ${ }^{1}$, T.C. Clancy $^{2}$, G.M. Odegard ${ }^{1}$, T.S. Gates ${ }^{3}$, E.C. Aifantis ${ }^{4}$ \\ ${ }^{1}$ Department of Mechanical Engineering-Engineering Mechanics, Michigan Technological University, Houghton, MI \\ ${ }^{2}$ National Institutive of Aerospace, Hampton, VA \\ ${ }^{3}$ NASA Langley Research Center, Hampton, VA \\ ${ }^{4}$ College of Engineering, Michigan Technological University, Houghton, MI
}

\begin{abstract}
A large number of possible polymer chain conformations exist for a given volume of an amorphous polymer. The prediction of elastic properties of a polymer must therefore consider more than a single combination of chain conformations. A multiscale modeling approach is proposed to predict the bulk elastic properties of polymer materials using a series of molecular models of individual polymer microstates and a statistics-based micromechanical modeling method. The method is applied to polyimide and polycarbonate systems. It is shown that individual microstates can yield a wide range of predicted elastic properties, whereas the consideration of multiple microstates yield predicted properties that more-closely agree with experimentally-determined values of Young's modulus. Additionally, the upper and lower limits of possible elastic constants are also established based on the consideration of multiple microstates.
\end{abstract}

Keywords: Molecular Dynamics, Mechanical Properties, Conformation Space, Multiscale Modeling

\section{Introduction}

Polymer-based nanocomposite materials have the potential to provide significant increases in specific stiffness and specific strength relative to current materials used for many engineering structural applications. The gains in mechanical properties are due to increases in the reinforcement surface area relative to conventional composite materials for a given reinforcement volume fraction. The increased reinforcement surface area results in significant increases in load transfer between the reinforcement and matrix phases. To facilitate the development of polymer nanocomposite materials, constitutive relationships must be established that predict the bulk mechanical properties of the materials as a function of the molecular structure and interactions.

Multiscale modeling techniques must be used to relate the molecular structure at the nanometer length-scale to mechanical behavior at the macro-length scale. Molecular Dynamics (MD) simulations can be used to predict the equilibrated molecular structures of polymer-based materials for a given thermodynamic state [1-8]. Also, the mechanical behavior of a molecular system can be studied when a representative volume element (RVE) of the molecular structure is subjected to applied deformations. While most multiscale modeling studies have focused on 
ordered crystalline materials, such as metals and ceramics [9-13], little attention has been given to multiscale modeling of amorphous materials, such as polymers and polymer nanocomposites.

Unlike crystalline materials, amorphous polymer materials contain an elaborate network of molecular chains with highly-complex and irregular conformations that dictate the bulk mechanical properties. Many combinations of the conformations of multiple polymer chains are possible for a particular RVE in an equilibrated or non-equilibrated state because of the finite entropy of the material for any temperature above $0 \mathrm{~K}$ owing to the dynamics of the constituent chains [2, 14-16]. As a result, the molecular structure, and thus density, of a polymer material varies substantially on the nanometer length-scale [16-23]. The large number of possible conformations for a specific volume of a polymer material constitutes a conformation space. Each combination of chain conformations in a RVE has an associated potential energy which can be interpreted as an energy landscape that depends on the conformational state of the polymer network. The conformational space does not necessarily have a one-to-one correspondence to the energy landscape. Therefore, the energy landscape generally consists of multiple local minima. As a result, for a RVE consisting of a finite number of polymer chains, there can exist multiple locally-equilibrated states.

A majority of high performance polymer-based materials operate at temperatures much below their glass transition temperatures. An amorphous polymeric material in a glassy state can be envisioned as a super-cooled liquid that is "frozen" in a local potential energy equilibrium state, which is not necessarily a globally-minimized potential energy state. The different microstates that are not at the global-minimum energy state are essentially "metastable" states with exceptionally long relaxation times as the energy barriers to cross over to the global minimum energy state are generally very high. Therefore, the bulk material behavior can be imagined to be an average response from all the available conformational microstates. In order to accurately predict the bulk-level behavior of polymer-based systems based on molecular structure, a range of conformational microstates of a polymeric network must be included in multiscale constitutive modeling approaches. In this study, a multiscale modeling technique was used to predict the bulk elastic moduli of a polyimide and a polycarbonate material system using multiple conformational states and statistical bounds of the predicted moduli are subsequently established. Physically-motivated statistically weighting of properties obtained from individual microstates for each polymer was incorporated into the modeling approach. It was found that the established bounds included experimentally-measured values of moduli for these materials. The framework of modeling presented here is very adaptable and can be extended to include any bulk physical property for an amorphous polymer material.

\section{Force Field}

Force fields are generally used in MD- and Molecular Mechanics (MM)-based atomistic simulations to describe the interactions between individual atoms and to relate the specific molecular configuration to the potential energy of a RVE of a material system. Force fields are generally semi-empirical and assume specific degrees of freedom for the constituent atomic structures. The total energy of the RVE of a molecular system is obtained as the summation of energies associated with individual degrees of freedom. One of the most widely-used force fields, AMBER, implemented as AMBER99 in the Tinker software package [24] was utilized for this study. The AMBER force field has a relatively simple functional form compared to other 
well-known force fields [25]. The simulations used in the current study did not include electrostatic interactions based on the dipole moment of the atoms. The AMBER force field was chosen over other available force fields of rather complex functional form for efficiency and accuracy. This topic has been previously studied in detail [8].

The total potential energy of a simulated molecular system computed with the AMBER force field is based on the summation of the bond-stretching, bond-bending, bond-torsion, and nonbonded energies given by

$$
\Lambda=\Lambda_{\text {stretch }}+\Lambda_{\text {bend }}+\Lambda_{\text {torsion }}+\Lambda_{\mathrm{nb}}
$$

where

$$
\begin{gathered}
\Lambda_{\text {stretch }}=\sum_{\text {stretch }} K_{r}\left(r-r_{\text {eq }}\right)^{2} \\
\Lambda_{\text {bend }}=\sum_{\text {bend }} K_{\theta}\left(\theta-\theta_{\text {eq }}\right)^{2} \\
\Lambda_{\text {torsion }}=\sum_{\text {torsion }}\left\{\frac{V_{1}}{2}[1+\cos (\phi+\zeta)]+\frac{V_{2}}{2}[1-\cos (2 \phi+\zeta)]+\frac{V_{3}}{2}[1+\cos (3 \phi+\zeta)]\right\} \\
\Lambda_{\mathrm{nb}}=\sum_{I<J} 4 \varepsilon_{I J}\left[\frac{\left(\sigma_{I J}\right)^{12}}{r_{I J}^{12}}-\frac{\left(\sigma_{I J}\right)^{6}}{r_{I J}{ }^{6}}\right]
\end{gathered}
$$

In Equations (1) - (5), the summations are taken over all of the corresponding interactions in the molecular model (RVE); $K_{r}$ and $K_{\theta}$ are the bond-stretching and bond-angle bending force constants, respectively; $r$ and $r_{e q}$ are the bond length and equilibrium bond lengths, respectively; $\theta$ and $\theta_{e q}$ are the bond angle and equilibrium bond angles, respectively; $V_{n} / 2, \zeta$, and $\phi$ are the torsion magnitude $(n=1,2,3)$, phase offset, and the torsion angle, respectively; and $\varepsilon_{I J}, r_{I J}$, and $\sigma_{I J}$ are van der Waals well depth, non-bonded distance between atoms $I$ and $J$, and the equilibrium distance between atoms $I$ and $J$, respectively. Because the functional form of the AMBER potential contains simplified harmonic terms for the bond stretching and bond-angle bending, it is expected to perform well only under relatively small deformations of the covalent bonds from their equilibrium state. Therefore, as described below, only relatively small deformations are used in this study.

\section{Molecular Modeling}

The simulations were carried out on both a polyimide and a polycarbonate system. These two polymers have been previously synthesized, tested, and modeled $[8,26,27]$. Figure 1 shows the chemical repeat structures of both polymers. Multiple RVEs representing samples from the conformational space were obtained for the two polymer materials. Nine thermally-equilibrated structures were obtained for polycarbonate, each consisting of 5958 atoms with 9 polymer chains and each chain comprised of 20 repeat units. Each of the nine resulting equilibrated structures 
represented a microstate for the polycarbonate system. For the polyimide system, seven locally equilibrated molecular structures of 6,622 atoms each were established with 11 chains and 10 repeat units per chain. Each of these seven polyimide structures represented a single microstate.

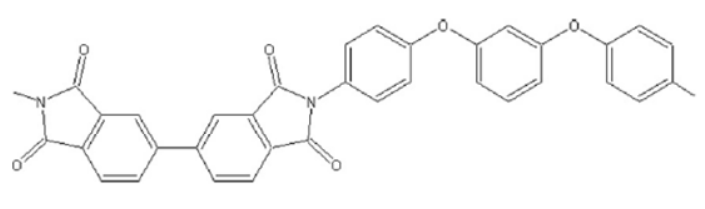

Polyimide
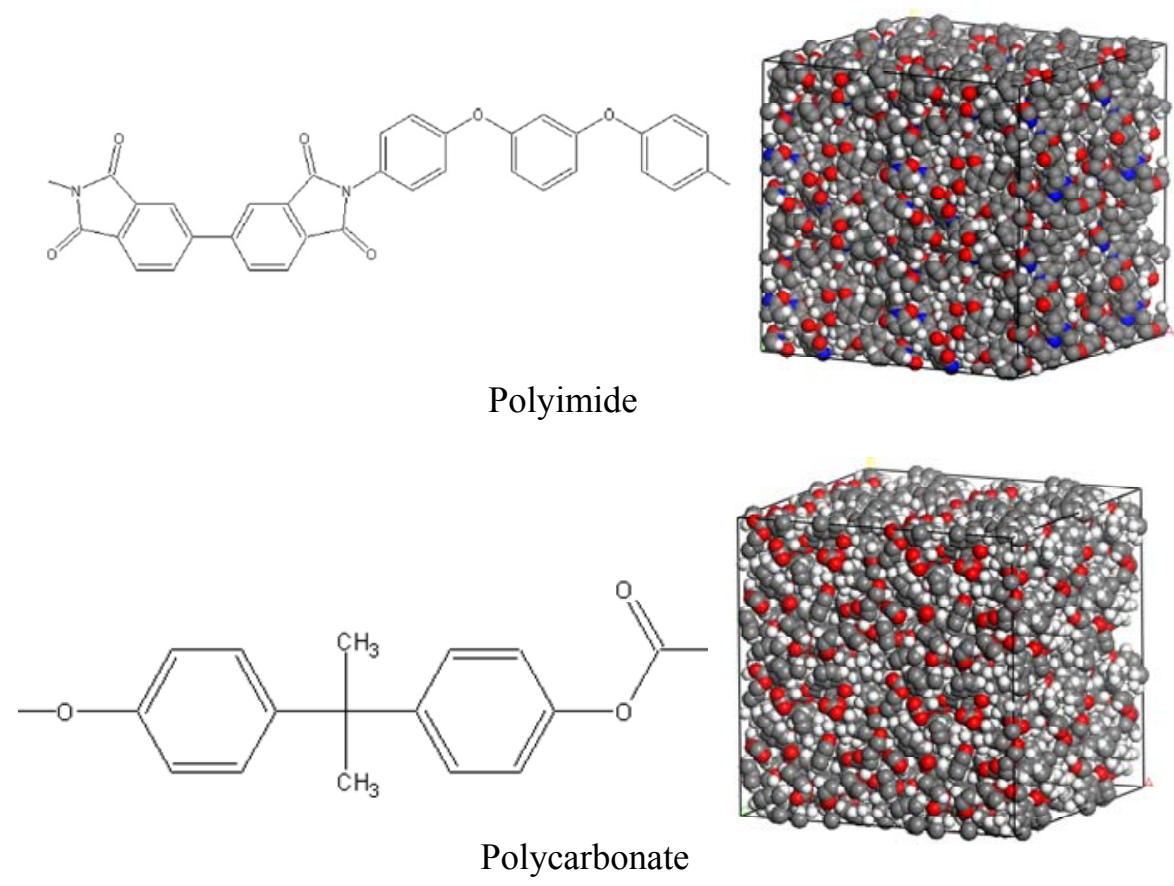

Figure 1. Schematic of the repeat unit for polymeric chains and the corresponding representative molecular model of the polymeric network

All RVE structures were initially prepared in a gas-like phase with extremely low densities. For each RVE sample, the polymer chains were placed in a simulation box with random conformations and orientations. Energy minimization simulations with periodic boundary conditions were conducted at gradually-increasing densities. The MINIMIZE [28] and NEWTON [29] subroutines of the TINKER [24] modeling package were used for the energy minimization, which correspond to a quasi-Newton L-BFGS method and a truncated Newton energy minimization methods, respectively. The minimizations were performed to RMS gradients of $1 \times 10^{-2}$ and $1 \times 10^{-5} \mathrm{kcal} / \mathrm{mole} / \AA \AA$, respectively.

Once the RVEs were established with the expected solid bulk density, a series of MD simulations were used to establish thermally-equilibrated solid structures in the following order at $300 \mathrm{~K}$ : (1) a 50 ps simulation with the NVT (constant number of atoms, volume, and temperature) ensemble to prepare the structure for further equilibration, (2) a 100 ps simulation with the NPT (constant number of atoms, pressure, and temperature) ensemble at 100 atm to evolve the system to higher densities as the structure was prepared from a low density structure, (3) a $100 \mathrm{ps}$ NPT simulation at $1 \mathrm{~atm}$ to reduce the effects of high-pressure simulations and to let the system evolve to a state of minimal residual stresses, and (4) a 100 ps NVT simulation to allow the system to equilibrate at the simulated temperature and density for a specific microstate. 
The DYANAMIC subroutine of the TINKER modeling package was used for the MD simulations with periodic boundary conditions. Examples of the molecular models that were established in a manner described above are shown in Figure 1.

\section{Equivalent-Continuum Properties}

To relate the molecular structure of the microstates of the polymer systems to their corresponding bulk mechanical properties, an equivalent-continuum modeling approach was used that effectively represented the mechanical behavior of the molecular RVEs. Because the molecular structures of the polymers were completely amorphous, it was assumed that the equivalent-continuum constitutive equation for the microstates exhibited isotropic symmetry. Based on this material symmetry for the equivalent continuum, a hyperelastic continuum constitutive relation [8] was used to model the deformation characteristics of the discrete molecular models. For generality, it was desired that the constitutive relationship of the equivalent continuum satisfy the following requirements: (1) formulated in a time-independent finite-deformation framework, (2) established using a thermodynamic potential, (3) incorporating isotropic material symmetry, and (4) expressed in terms of volumetric (shape preserving) and isochoric (shape changing) contributions. The assumed form of the equivalentcontinuum strain energy is

$$
\Psi_{c}=\psi_{\text {vol }}+\psi_{\text {iso }}
$$

where

and

$$
\begin{aligned}
& \psi_{\text {vol }}=c_{1} \Omega_{1} \\
& \psi_{\text {iso }}=c_{2} \Omega_{2}
\end{aligned}
$$

$$
\Omega_{1}=\left(I_{3}-1\right)^{2} \quad \Omega_{2}=\left(\frac{I_{1}}{I_{3}^{1 / 3}}+\frac{I_{2}^{3}}{I_{3}^{2}}-30\right)
$$

The parameters $\Omega_{1}$ and $\Omega_{2}$ in Equation (8) represent the volumetric and isochoric components of the strain-energy density; $c_{1}$ and $c_{2}$ are constants which represent material properties; and $I_{1}$, $I_{2}$, and $I_{3}$ are the scalar invariants of the right Cauchy-Green deformation tensor, $\mathbf{C}$. The second Piola-Kirchhoff stress tensor is therefore [8]

$$
\mathbf{S}=\frac{2}{3}\left[6 c_{1} I_{3}\left(I_{3}-1\right)-c_{2}\left(\frac{I_{1}}{I_{3}^{1 / 3}}+6 \frac{I_{2}{ }^{3}}{I_{3}{ }^{2}}\right)\right] \mathbf{C}^{-1}+2 c_{2}\left(\frac{1}{I_{3}{ }^{1 / 3}}+3 \frac{I_{1} I_{2}{ }^{2}}{I_{3}{ }^{2}}\right) \mathbf{I}-6 c_{2} \frac{I_{2}{ }^{2}}{I_{3}{ }^{2}} \mathbf{C}
$$

where $\mathbf{I}$ is the identity tensor. It has been shown that any hyperelastic constitutive equation, such as Equation (9), can be reduced to the linearized Hooke's law for small deformations (see, for example, reference [30] page 283 or reference [31] page 187). Therefore, the constitutive law of Equation (9) can be expressed in terms of the classic elastic stiffness constants and the Young's modulus for infinitesimal deformations. 
Equation (9) contains material parameters $c_{1}$ and $c_{2}$ which were evaluated for each microstate by equating the equivalent-continuum strain energy and the molecular potential energy for a set of identical deformation fields applied to the equivalent continuum and the molecular models, as has been performed in previous studies of other nanostructured materials [4, 8, 32, 33]. The molecular potential energy was calculated for each deformation using molecular statics. Specifically, for each deformation, an energy minimization was performed with periodic boundary conditions. The MINIMIZE [28] and NEWTON [29] subroutines of the TINKER [24] modeling package were used for the energy minimization, which correspond to a quasi-Newton L-BFGS method and a truncated Newton energy minimization methods, respectively. The minimizations were performed to RMS gradients of $1 \times 10^{-2}$ and $1 \times 10^{-5} \mathrm{kcal} / \mathrm{mole} / \AA$, respectively. For the molecular models, the strain energy densities were computed from the force fields using

$$
\Psi_{m}=\frac{1}{V_{0}}\left(\Lambda_{m}-\Lambda_{m}^{0}\right)
$$

where $\Lambda_{m}^{0}$ and $\Lambda_{m}$ are the molecular potential energies in the undeformed state and after application of the deformations, respectively, which were directly computed from the force field; and $V_{0}$ is the volume of the simulation box in the undeformed state.

The molecular models of the polymer systems were subjected to two different deformation fields, a pure volumetric deformation and an isochoric deformation. Finite deformations were applied to the molecular and equivalent-continuum models in incremental steps. For the volumetric deformation, volumetric strains $\left(E_{11}=E_{22}=E_{33}\right.$ where $\mathbf{E}$ is the Green strain tensor) of $0.1 \%, 0.2 \%, 0.3 \%, 0.4 \%$ and $0.5 \%$ were applied. For the isochoric deformations, threedimensional shear strain levels of $\gamma_{23}=\gamma_{13}=\gamma_{12}=0.1 \%, 0.2 \%, 0.3 \%, 0.4 \%$ and $0.5 \%\left(\gamma_{i j}=2 E_{i j}\right.$ when $i \neq j$ ) were applied. The volumetric and isochoric deformations were used to determine the bulk moduli $K$ and shear moduli $\mu$, respectively, for each microstate of the two polymer systems. The components of the stiffness tensor $\mathbf{L}$ were determined for each microstate using

$$
L_{i j k l}=K \delta_{i j} \delta_{k l}+\mu\left(\delta_{i k} \delta_{j l}+\delta_{i l} \delta_{j k}-\frac{2}{3} \delta_{i j} \delta_{k l}\right)
$$

Further details on these deformations and modulus calculations can be found elsewhere [8]. Regarding the assumption of material isotropy, the total magnitudes of deformation are relatively small, therefore, any strain-induced anisotropy, which is usually observed at much larger values of strains ( $>10 \%$ strain), is neglected. Elastic constants, such as Young's modulus, can be directly determined from the stiffness tensor of a material using Equation (11) and the relationships between elastic constants of isotropic materials [30].

At this point, a brief discussion about the Young's modulus calculated statically is necessary. With the static approach used above (with static molecular equilibrations of the molecular structure), the Young's modulus is estimated based on the material behavior over an ambiguous time interval associated with the molecular equilibration. The time-based ambiguity is due to the energy equilibration of the molecular structure irrespective of a simulated time. Time-dependent 
(viscoelastic) responses in thermoplastic polymers are beyond the reach of time scales that are achievable with current time-stepping schemes using MD approaches $\left(1 \times 10^{15}\right.$ simulated time steps for a fully atomistic model). Therefore, static approaches efficiently simulate deformation at relatively large, yet quantitatively undefined time intervals that are realistic for engineering experience. Because thermoplastic polymers exhibit time-dependent behavior, it is expected that static approach used herein should yield Young's moduli that are directly analogous to the Young's moduli that are typically measured for thermoplastics in a laboratory setting with tests that are run on the order of minutes. The results of a previous study [8] reinforce this expectation. When mechanical properties of viscoelastic polymers were estimated with dynamic deformation techniques, which were limited to picoseconds time scales using the available computation resources, large over-estimations of the Young's moduli with respect to experimentally-obtained values were predicted [8]. These large over-predictions were avoided using static techniques [8]. The issue of using static minimizations instead of dynamic-based simulations to establish material properties has also been addressed by other researchers [34].

\section{Micromechanics}

Because a given RVE has a unique combination of chain conformations, it is expected that the above-discussed approach to predicting the elastic properties of a polymer system will generally yield different predicted properties for different nanometer-scale RVEs. It is also expected that the bulk material response will be dependent on the mechanical response of all such microstates that are possible for a given polymer system. Because of the computational difficulty of establishing every possible microstate for a molecular RVE of a polymer system, the modeling procedure described herein is restricted to the finite number of microstate RVEs obtained as described above.

Micromechanics-based techniques [35] were used to determine the equivalent bulk-level response of the two polymer systems based on the mechanical response of the microstates. This section describes the use of these techniques to establish the bulk properties and the upper and lower bounds of possible bulk properties for the two polymer systems.

\section{Equivalent Bulk Property Bounds}

Upper and lower bounds of elastic constants of composite materials are often established to set the boundaries of possible predicted properties predicted with micromechanical approaches. If it is assumed that the strains of all of the phases of a composite material are the same for a given bulk-level deformation, the resulting Voigt model predicts the upper bound of possible bulk composites elastic properties [35]. If it is assumed that the phases of a composite material have the same stress components for a given bulk-level deformation, the resulting Reuss model serves as a lower bound of possible bulk composite properties. The Voigt and Reuss models are given by, respectively,

$$
\mathbf{L}^{V}=\sum_{r=0}^{N} c_{r} \mathbf{L}_{r}
$$


Acta Materialia, Vol. 57, no. 2, pp. 525-532 (2009)

$$
\mathbf{L}^{R}=\left[\sum_{r=0}^{N} c_{r} \mathbf{M}_{r}\right]^{-1}
$$

where $\mathbf{L}^{V}$ and $\mathbf{L}^{R}$ are the effective stiffness tensor representing Voigt and Ruess bounds, respectively; $\mathbf{L}_{r}$ and $\mathbf{M}_{r}$ are the stiffness and compliance tensor components of phase $r$, respectively; $N$ is the total number of microstates considered; and $c_{r}$ is the volume fraction of phase $r$ where

$$
\sum_{r=0}^{N} c_{r}=1
$$

Although tighter bounds have been established for composite materials [36], these bounds assume specific geometries of the phases, such as fibrous or spherical reinforcements. Because the phases considered herein do not necessarily have a defined geometric shape, Equations (12) and (13) are assumed to be the bounds for the bulk mechanical properties of the two polymer systems.

\section{Equivalent bulk elastic properties}

While the above-discussed upper and lower bounds of stiffness tensor components provide the limits of the overall bulk properties, it is useful for material design purposes to use micromechanical techniques to predict an accurate estimate of bulk-level properties. The vast majority of micromechanical techniques focus on predicting properties of composite materials with well-defined reinforcement geometry [35]. However, the geometry of simulated microstates within the polymer material is unknown. Therefore, it was assumed that the bulk polymer stiffness tensor $\mathbf{L}$ is determined using the following simple rule-of-mixtures equation

$$
\mathbf{L}=\sum_{r=0}^{N} c_{r} \mathbf{L}_{r}
$$

It is important to note that the form of Equation (15) is the same as that of Voigt model, Equation(12). The form of Equation (15) was chosen because of its simplicity. Therefore, the simplest estimates of the bulk mechanical properties will coincide with the upper bound of possible predicted properties of the bulk polymer.

\section{Energy-based Statistics}

Both the Voigt-Reuss bounds and predicted bulk properties are dependent on the volume fractions of the constituent microstates, as indicated by Equations (12), (13), and (15). However, because the distribution of microstates in a polymer material is not always known, simple approaches for selecting the relative volume fractions of the phases must be established. If it is assumed that the volume fraction of a particular microstate is equal to the probability of the existence of that same microstate, then $c_{r}$ in Equation (15) can be replaced by the probability 
$p_{r}$. Because there are no well-established distribution functions that describe $p_{r}$ for a polymer material, three assumed forms of the function were considered for this study.

The first approach is to assume that all the microstates of the polymer are statistically equally likely, which leads to same volume fractions for each microstate. Therefore, the resulting probability of a microstate $r$ existing in a sample of a polymer material is

$$
p_{r}^{(1)}=\frac{1}{N}
$$

where $N$ is the total number of different microstates considered, and the superscript 1 indicates the probability associated with this first assumption. The definition in Equation (16) satisfies the requirement that

$$
\sum_{r=1}^{N} p_{r}^{(1)}=1
$$

Although this approach is very simple, a second approach to determining $p_{r}$ is to use a physically-intuitive probability distribution that is dependent on the energy of a particular microstate. Boltzmann statistics are widely-used to determine the probability of a particular microstate [37]. The service temperature of most engineering polymers is much below the glass transition temperature, and thus many polymers are in a glassy state. Due to the statistical nature of the growth of polymer networks during the synthesis of addition polymers [38], the networks do not crystallize and the chain dynamics are significantly hindered due to the formation of elaborate entanglements. The entanglements resist the free movement of the polymer chains and therefore hinder the network evolution to a globally-minimized potential energy state. Therefore, it is expected that the lowest-energy microstates are more common, with a finite number of higher-energy microstates. Motivated by the second law of thermodynamics, a second probability distribution function is assumed that favors lower-energy microstates over the higher-energy microstates which results in an energy-biased distribution

$$
p_{r}^{(2)}=\frac{\Lambda_{r}^{-1}}{\sum_{s=1}^{N}\left(\Lambda_{s}\right)^{-1}}
$$

where $\Lambda_{r}$ is the potential energy of microstate $r$ calculated using Equations (1)-(5). In a similar manner, a third probability distribution function is assumed to be

$$
p_{r}^{(3)}=\frac{\Lambda_{r}^{-2}}{\sum_{s=1}^{N}\left(\Lambda_{s}\right)^{-2}}
$$


The probability distributions described by Equations (18) and (19) obey the normalization condition of Equation (17). The functional forms of Equations (18) and (19) clearly assign higher probabilities to microstates with lower energies.

Table 1. Polyimide microstate properties

\begin{tabular}{|c|c|c|c|c|c|c|c|c|}
\hline Microstate & $\begin{array}{c}\Lambda_{r} \\
(\mathrm{kcal} / \mathrm{mole})\end{array}$ & $\begin{array}{c}\text { Simulation } \\
\text { box size } \\
(\AA)\end{array}$ & $\begin{array}{c}\text { Density } \\
(\mathrm{g} / \mathrm{cc})\end{array}$ & $\begin{array}{c}\text { Young's } \\
\text { modulus } \\
(\mathrm{GPa})\end{array}$ & $\begin{array}{c}\text { Shear } \\
\text { modulus } \\
(\mathrm{GPa})\end{array}$ & $p_{r}^{(1)}$ & $p_{r}^{(2)}$ & $p_{r}^{(3)}$ \\
\hline 1 & $42,788.42$ & 44.31 & 1.16 & 0.02 & 0.01 & 0.143 & 0.233 & 0.345 \\
\hline 2 & $52,780.01$ & 44.29 & 1.16 & 0.24 & 0.08 & 0.143 & 0.189 & 0.227 \\
\hline 3 & $79,859.22$ & 44.46 & 1.14 & 4.71 & 1.64 & 0.143 & 0.125 & 0.099 \\
\hline 4 & $84,264.01$ & 44.76 & 1.12 & 11.10 & 4.14 & 0.143 & 0.118 & 0.089 \\
\hline 5 & $84,682.77$ & 44.94 & 1.11 & 3.82 & 1.31 & 0.143 & 0.118 & 0.088 \\
\hline 6 & $85,469.25$ & 44.87 & 1.11 & 10.20 & 3.66 & 0.143 & 0.116 & 0.086 \\
\hline 7 & $97,465.05$ & 44.55 & 1.14 & 16.70 & 6.44 & 0.143 & 0.102 & 0.066 \\
\hline
\end{tabular}

Table 2. Polycarbonate microstate properties

\begin{tabular}{|c|c|c|c|c|c|c|c|c|}
\hline Microstate & $\begin{array}{c}\Lambda_{r} \\
(\mathrm{kcal} / \mathrm{mole})\end{array}$ & $\begin{array}{c}\text { Simulation } \\
\text { box size } \\
(\AA)\end{array}$ & $\begin{array}{c}\text { Density } \\
(\mathrm{g} / \mathrm{cc})\end{array}$ & $\begin{array}{c}\text { Young's } \\
\text { modulus } \\
(\mathrm{GPa})\end{array}$ & $\begin{array}{c}\text { Shear } \\
\text { modulus } \\
(\mathrm{GPa})\end{array}$ & $p_{r}^{(1)}$ & $p_{r}^{(2)}$ & $p_{r}^{(3)}$ \\
\hline 1 & $13,706.69$ & 41.21 & 1.09 & 2.29 & 0.78 & 0.111 & 0.175 & 0.232 \\
\hline 2 & $13,722.27$ & 40.77 & 1.12 & 5.14 & 1.77 & 0.111 & 0.175 & 0.231 \\
\hline 3 & $15,905.73$ & 41.38 & 1.07 & 2.01 & 0.68 & 0.111 & 0.151 & 0.172 \\
\hline 4 & $18,093.05$ & 40.90 & 1.11 & 12.00 & 4.21 & 0.111 & 0.133 & 0.133 \\
\hline 5 & $21,674.48$ & 40.49 & 1.15 & 20.90 & 7.65 & 0.111 & 0.111 & 0.093 \\
\hline 6 & $23,372.80$ & 40.91 & 1.11 & 0.09 & 0.03 & 0.111 & 0.103 & 0.080 \\
\hline 7 & $38,850.61$ & 40.86 & 1.12 & 5.69 & 1.95 & 0.111 & 0.062 & 0.029 \\
\hline 8 & $50,629.76$ & 41.18 & 1.09 & 7.08 & 2.44 & 0.111 & 0.047 & 0.017 \\
\hline 9 & $55,728.69$ & 41.94 & 1.03 & 1.65 & 0.56 & 0.111 & 0.043 & 0.014 \\
\hline
\end{tabular}

\section{Results}

Tables 1 and 2 show the results obtained from the MD simulations of each microstate of the polyimide and polycarbonate materials, respectively. The results are arranged in the increasing order of the equilibrium energies. From these tables, it is clear that the equilibrium energies of the microstates varied greatly for both polymers. The variation in microstate energies confirms the assumption that the conformational space accessible to the polymeric chain network is extensive and the current modeling techniques sample a very small subset of the metastable potential energy states that exist in local equilibrium. The densities of the polyimide ranged from $1.11 \mathrm{~g} / \mathrm{cc}$ to $1.16 \mathrm{~g} / \mathrm{cc}$ with a mean of $1.13 \mathrm{~g} / \mathrm{cc}$. The densities of the polycarbonate ranged from $1.03 \mathrm{~g} / \mathrm{cc}$ to $1.15 \mathrm{~g} / \mathrm{cc}$ with an average of $1.10 \mathrm{~g} / \mathrm{cc}$. Therefore, the densities obtained from these simulations were very consistent among the different microstate of both polymers. Similarly, the 
equilibrium RVE cubic side dimensions varied little between the microstates of the polymer systems.

Tables 1 and 2 also list the Young's and shear moduli determined for each microstate using the above-described method. The calculated Young's moduli for polyimide varied from $0.02-16.70$ $\mathrm{GPa}$, with an average of $6.68 \mathrm{GPa}$ and a standard deviation of $6.19 \mathrm{GPa}$. In case of polycarbonate the Young's modulus varied from 0.09 to $20.90 \mathrm{GPa}$ with an average of $6.32 \mathrm{GPa}$ and a standard deviation of $6.54 \mathrm{GPa}$. For both the polymer materials the standard deviations are on the order of the averages of the Young's moduli. A similar trend exists for the predicted shear moduli of the two polymer systems, as shown in Tables 1 and 2 . The large standard deviations in the predicted elastic properties are a consequence of the limited number of samples used in the study and the limited size of the RVE. Because of computational costs, the number and size of RVEs was limited in this study and significant standard deviations in the predicted properties were expected. Tables 1 and 2 also list the resulting values of $p_{r}^{(1)}, p_{r}^{(2)}$, and $p_{r}^{(3)}$ for each microstate of both polymers. Clearly the probability distribution is more skewed toward lower energy values for $p_{r}^{(2)}$ and $p_{r}^{(3)}$ for both polymers. Furthermore, $p_{r}^{(3)}$ has the highest probabilities at the lowest energies, and the lowest probabilities at the highest energies relative to $p_{r}^{(1)}$ and $p_{r}^{(2)}$ for both polymers.

Table 3. Young's modulus values of polymer systems

\begin{tabular}{|c|c|c|c|c|}
\hline Material & $\begin{array}{c}\text { Young's } \\
\text { modulus from } \\
\text { experiment } \\
(\mathrm{GPa})\end{array}$ & $\begin{array}{c}\text { Young's } \\
\text { modulus from } \\
p_{r}^{(1)} \text { distribution } \\
(\mathrm{GPa})\end{array}$ & $\begin{array}{c}\text { Young's } \\
\text { modulus from } \\
p_{r}^{(2)} \text { distribution } \\
(\mathrm{GPa})\end{array}$ & $\begin{array}{c}\text { Young's } \\
\text { modulus from } \\
p_{r}^{(3)} \text { distribution } \\
(\mathrm{GPa})\end{array}$ \\
\hline Polyimide & 3.90 & 6.86 & 5.42 & 3.93 \\
\hline Polycarbonate & 2.20 & 6.41 & 6.38 & 6.02 \\
\hline
\end{tabular}

Table 3 shows the predicted bulk Young's modulus values for both polymer systems for each of the three microstate probability distribution functions. In addition, experimentally-obtained Young's modulus values from the literature [26, 39] have also been listed in Table 3. For the polyimide, the predicted value of Young's modulus for the $p_{r}^{(3)}$ probability distribution is the lowest of the three distribution functions. The predicted Young's modulus from the $p_{r}^{(1)}$ distribution were the highest relative to the other distribution functions and showed the least agreement with the experimentally-obtained Young's modulus. The same trend is observed for the predicted moduli of the polycarbonate. However, the predicted polyimide Young's modulus from the $p_{r}^{(3)}$ distribution matched the experimental value very closely while the polycarbonate Young's modulus from the same distribution function is significantly higher than the experimental value.

Because the micromechanical model that was used to predict the elastic response of the polymers [Equation (15)] is a simple approximation, the Voigt-Reuss bounds provide a set of limits that the bulk Young's modulus is physically constrained to based entirely on mechanical interactions between the microstates. Figures 2 and 3 are linear-elastic uniaxial stress-strain 
curves for the polyimide and polycarbonate systems, respectively. On both graphs appear the Voigt-Reuss bounds determined with Equation (12)-(13) for each of the three probability distribution functions $p_{r}^{(1)}, p_{r}^{(2)}$, and $p_{r}^{(3)}$. Also shown in Figures 2 and 3 are the polymer responses as determined from experiments [26, 39]. For the polyimide, the experimental data falls between the upper and lower bounds, and matches the upper bound of the response determined with $p_{r}^{(3)}$. For the polycarbonate system, the experimental curve appears to be nearly half-way between the Voigt and Reuss bounds.

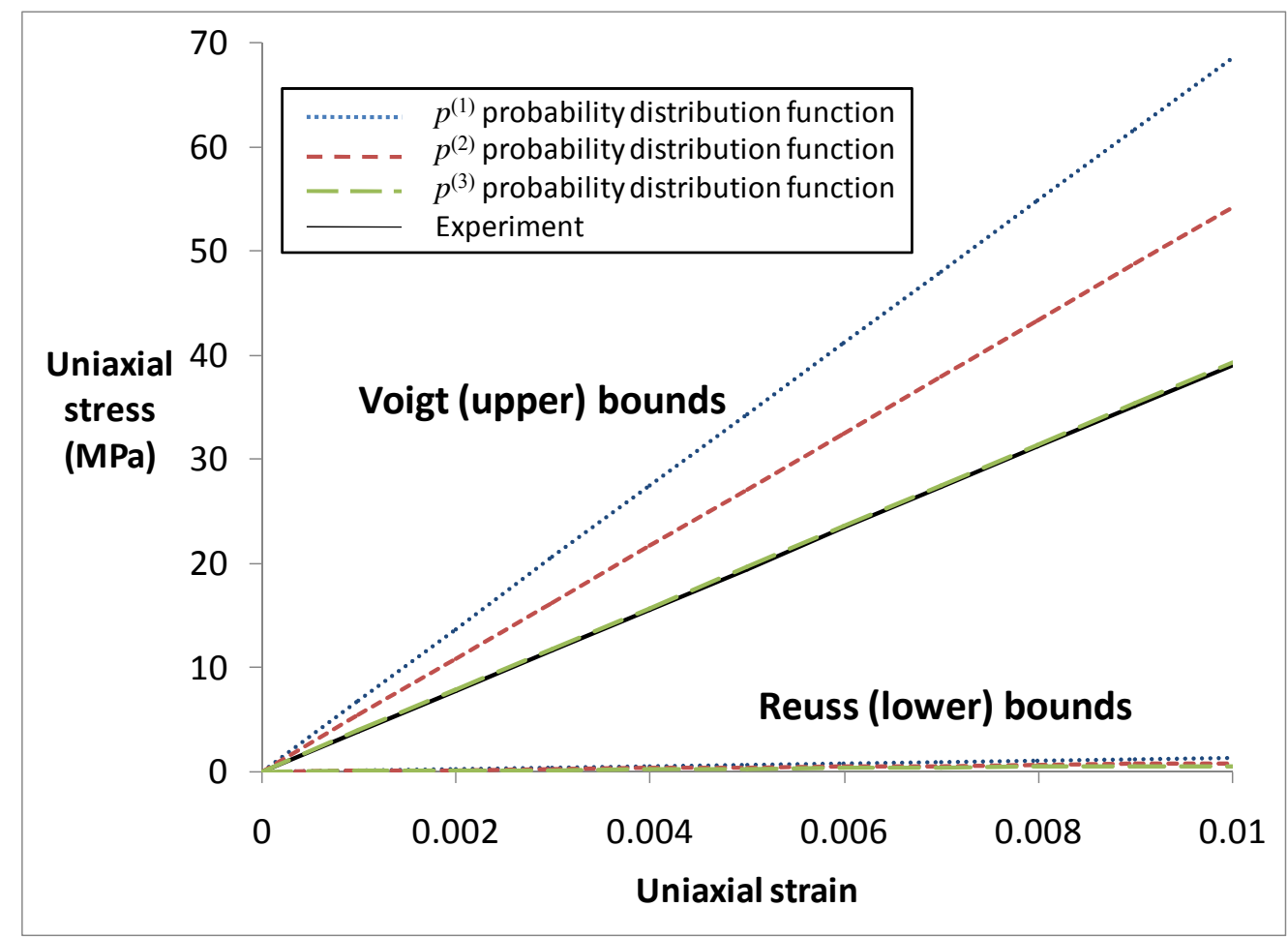

Figure 2. Bounds for Young's modulus of polyimide

\section{Conclusions}

The bulk elastic properties of a polyimide system and a polycarbonate polymer have been predicted based on the molecular structure of several microstate representative volume elements whose cubic side dimensions are on the order of a few nanometers. A micromechanical approach has been used to predict the bulk properties based on the predicted mechanical response of each microstate for both polymer systems. The theoretical bounds of possible predicted properties have also been established. The results indicate that individual microstates can have a wide range of Young's moduli, differing by as much as $16.7 \mathrm{GPa}$ for the polyimide and $21.8 \mathrm{GPa}$ for the polycarbonate. These differences are a factor of 4 and 10, respectively, higher than the magnitude of the experimentally-obtained values of Young's modulus from the literature. On the other hand, the proposed statistics-based modeling approach has yield predicted bulk Young's modulus values that are higher than the experimental values by a factor of 1 to 3 , depending on the assumed probability distribution function. Therefore, the 
consideration of multiple microstates for a polymer is necessary for the multiscale prediction of elastic properties based on molecular structure.

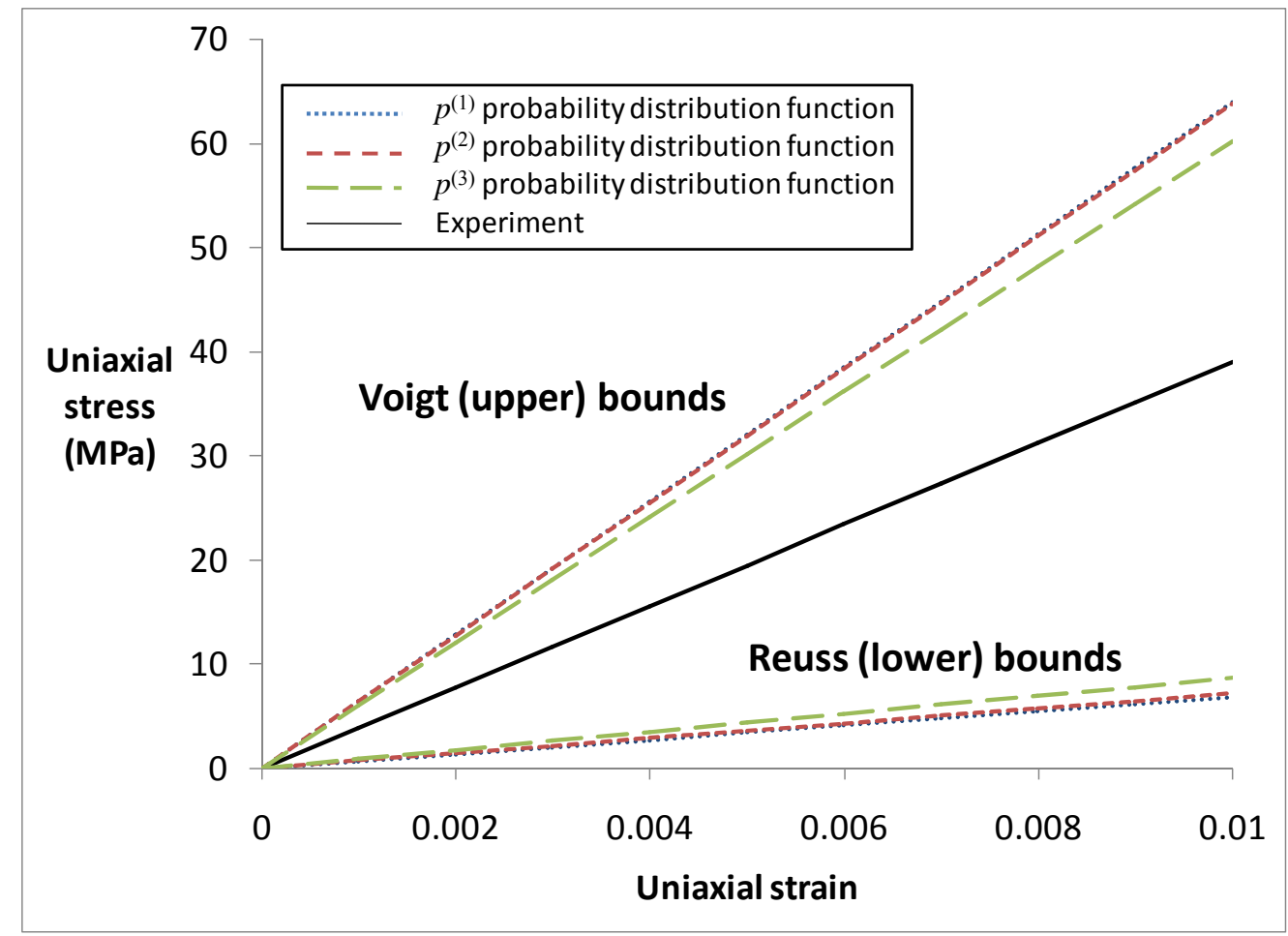

Figure 3. Bounds of Young's modulus of polycarbonate

Although the predicted Young's moduli of both polymers systems are higher than the experimental values for all three distribution functions proposed, an over-prediction of elastic properties is expected for two reasons. First, the molecular systems modeled in the current study represent polymer structures without any chain length distribution and unreacted monomer, both of which are expected to reduce the overall elastic properties of a polymer. Therefore, the predicted properties from these models are expected to be higher than those experimentallyobserved in the laboratory. Second, the proposed micromechanics model functional form is a simple rule-of-mixtures formulation. The form of the model is identical with the upper-bound of possible elastic properties. Therefore, a more realistic, and possibly more complex, micromechanics model will likely predict bulk Young's modulus values that are closer to experiment than those presented herein.

Acknowledgements. This research was jointly sponsored by NASA Langley Research Center under grant NNL04AA85G and the National Science Foundation under grant DMI-0403876. The authors also acknowledge the helpful discussions with Dr. Walter Nadler. 
Acta Materialia, Vol. 57, no. 2, pp. 525-532 (2009)

\section{References}

[1] Brown D, Clarke JHR. Molecular-Dynamics Simulation of an Amorphous Polymer Under Tension. 1. Phenomenology. Macromolecules 1991;24:2075.

[2] Capaldi FM, Boyce MC, Rutledge GC. Molecular response of a glassy polymer to active deformation. Polymer 2004;45:1391.

[3] Frankland SJV, Caglar A, Brenner DW, Griebel M. Molecular Simulation of the Influence of Chemical Cross-Links on the Shear Strength of Carbon Nanotube-Polymer Interfaces. Journal of Physical Chemistry B 2002;106:3046.

[4] Frankland SJV, Harik VM, Odegard GM, Brenner DW, Gates TS. The Stress-Strain Behavior of Polymer-Nanotube Composites from Molecular Dynamics Simulation. Composites Science and Technology 2003;63:1655.

[5] Hoy RS, Robbins MO. Strain hardening of polymer glasses: Effect of entanglement density, temperature, and rate. Journal of Polymer Science Part B-Polymer Physics 2006;44:3487.

[6] Lyulin AV, Li J, Mulder T, Vorselaars B, Michels MAJ. Atomistic simulation of bulk mechanics and local dynamics of amorphous polymers. Macromolecular Symposia 2006;237:108.

[7] Theodorou DN. Understanding and predicting structure-property relations in polymeric materials through molecular simulations. Molecular Physics 2004;102:147.

[8] Valavala PK, Clancy TC, Odegard GM, Gates TS. Nonlinear Multiscale Modeling of Polymer Materials. International Journal of Solids and Structures 2007;44:1161.

[9] de la Rubia TD, Zbib HM, Khraishi TA, Wirth BD, Victoria M, Caturla MJ. Multiscale modelling of plastic flow localization in irradiated materials. Nature 2000;406:871.

[10] Szlufarska I, Nakano A, Vashishta P. A crossover in the mechanical response of nanocrystalline ceramics. Science 2005;309:911.

[11] Wendel JA, Goddard WA. The Hessian Biased Force-Field for Silicon-Nitride Ceramics - Predictions of Thermodynamic and Mechanical-Properties for Alpha-Si3N4. Journal of Chemical Physics 1992;97:5048.

[12] Wu HA, Liu GR, Wang JS. Atomistic and continuum simulation on extension behaviour of single crystal with nano-holes. Modelling and Simulation in Materials Science and Engineering 2004;12:225.

[13] Yamakov V, Wolf D, Phillpot SR, Mukherjee AK, Gleiter H. Deformation-mechanism map for nanocrystalline metals by molecular-dynamics simulation. Nature Materials 2004;3:43.

[14] Laot CM, Marand E, Schmittmann B, Zia RKP. Effects of cooling rate and physical aging on the gas transport properties in polycarbonate. Macromolecules 2003;36:8673.

[15] Lyulin AV, Michels MAJ. Molecular dynamics simulation of bulk atactic polystyrene in the vicinity of T-g. Macromolecules 2002;35:1463.

[16] Stachurski ZH. Strength and deformation of rigid polymers: structure and topology in amorphous polymers. Polymer 2003;44:6059.

[17] Cohen MH, Turnbull D. On the Free-volume Model of Liquid-Glass Transition. Journal of Chemical Physics 1970;52:3038.

[18] Dlubek G, Pointeck J, Shaikh MQ, Hassan EM, Krause-Rehberg R. Free volume of an oligomeric epoxy resin and its relation to structural relaxation: Evidence from positron lifetime and pressure-volume-temperature experiments. Physical Review E 2007;75.

[19] Hinkley JA, Eftekhari A, Crook RA, Jensen BJ, Singh JJ. Free-Volume in Glassy Poly(arylene ether ketone)s. Journal of Polymer Science Part B-Polymer Physics 1992;30:1195. 
[20] Roe RJ, Curro JJ. Small-Angle X-Ray-Scattering Study of Density Fluctuation in Polystyrene Annealed Below the Glass-Transition Temperature. Macromolecules 1983;16:428.

[21] Roe RJ, Rigby D. Free-Volume Distribution and Local Chain Motion in Polymer Liquid and Glass Studied by MD-Simulation. Abstracts of Papers of the American Chemical Society 1990;199:361.

[22] Rutledge GC, Suter UW. Calculation of Mechanical-Properties of Poly(P-phenylene terephthalamide) by Atomistic Modeling. Polymer 1991;32:2179.

[23] Wilks BR, Chung WJ, Ludovice PJ, Rezac ME, Meakin P, Hill AJ. Structural and freevolume analysis for alkyl-substituted palladium-catalyzed poly(norbornene): A combined experimental and Monte Carlo investigation. Journal of Polymer Science Part B-Polymer Physics 2006;44:215.

[24] Ponder JW. TINKER - Software Tools for Molecular Design. St. Louis, MO: Washington University School of Medicine, 2004.

[25] Weiner PK, Kollman PA. AMBER - Assisted Model-Building with Energy Refinement A General Program for Modeling Molecules and their Interactions. Journal of Computational Chemistry 1981;2:287.

[26] Hergenrother PM, Watson KA, Smith JG, Connell JW, Yokota R. Polyimides from 2,3,3',4'-Biphenyltetracarboxylic Dianhydride and Aromatic Diamines. Polymer 2002;43:5077.

[27] Clancy TC. Multi-Scale Modeling of Polyimides. Polymer 2004;45:7001.

[28] Nocedal J, Wright SJ. Numerical Optimization. New York: Springer-Verlag, 1999.

[29] Ponder JW, Richards FM. An Efficient Newton-Like Methods for Molecular Mechanics Energy Minimization of Large Molecules. Journal of Computational Chemistry 1987;8:1016.

[30] Malvern LE. Introduction to the Mechanics of a Continuous Medium. Upper Saddle River, NJ: Prentice-Hall, Inc., 1969.

[31] Eringen AC. Mechanics of Continua. New York: John Wiley \& Sons, Inc., 1967.

[32] Theodorou DN, Suter UW. Atomistic Modeling of Mechanical Properties of Polymeric Glasses. Macromolecules 1986;19:139.

[33] Van Workum K, de Pablo JJ. Computer Simulation of the Mechanical Properties of Amorphous Polymer Nanostructures. Nano Letters 2003;3:1405.

[34] Payne MC, Teter MP, Allan DC, Arias TA, Joannopoulos JD. Iterative Minimization Techniques for Ab Initio Total-Energy Calculations - Molecular Dynamic and Conjugate Gradients. Reviews of Modern Physics 1992;64:1045.

[35] Qu J, Cherkaoui M. Fundamentals of Micromechanics of Solids. New York: Wiley, 2006.

[36] Hashin Z, Shtrikman S. A variational approach to the theory of elastic behaviour of multiphase materials. Journal of the Mechanics and Physics of Solids 1963;11:127.

[37] Hill TL. An Introduction of Statistical Thermodynamics. Mineola, NY: Dover Publications, 1986.

[38] Odian G. Principles of Polymerization. New York: Wiley-Interscience, 1991.

[39] Christopher WF, Fox DW. Polycarbonates. New York: Reinhold Publishing Corporation, 1962. 